\title{
Real-Time Coupling of Geographically Distributed Research Infrastructures: Taxonomy, Overview and Real-World Smart Grid Applications
}

\author{
IEEE-PES Task Force on Interfacing Techniques for Simulation Tools
}

\author{
M. H. Syed, Member, IEEE, E. Guillo-Sansano, Member, IEEE, Y. Wang, Member, IEEE, S. Vogel, Student \\ Member, IEEE, P. Palensky, Senior Member, IEEE, G. M. Burt, Member, IEEE, Y. Xu, Senior Member, IEEE, \\ A. Monti, Senior Member, IEEE, R. Hovsapian
}

\begin{abstract}
Novel concepts enabling a resilient future power system and their subsequent experimental evaluation are experiencing a steadily growing challenge: large scale complexity and questionable scalability. The requirements on a research infrastructure (RI) to cope with the trends of such a dynamic system therefore grow in size, diversity and costs, making the feasibility of rigorous advancements questionable by a single RI. Analysis of large scale system complexity has been made possible by the real-time coupling of geographically separated RIs undertaking geographically distributed simulations (GDS), the concept of which brings the equipment, models and expertise of independent RIs, in combination, to optimally address the challenge. This paper presents the outputs of IEEE PES Task Force on Interfacing Techniques for Simulation Tools towards standardization of GDS as a concept. First, the taxonomy for setups utilized for GDS is established followed by a comprehensive overview of the advancements in real-time couplings reported in literature. The overview encompasses fundamental technological design considerations for GDS. The paper further presents four application oriented case studies (real-world implementations) where GDS setups have been utilized, demonstrating their practicality and potential in enabling the analysis of future complex power systems.
\end{abstract}

\section{INTRODUCTION}

The transition of the electrical power grid from centralised operation to a more decentralised and distributed operating regime presents urgent and critical research challenges. For example, within the next decade, the Great Britain (GB) power system is projected to accommodate approximately 600,000 controllable distributed generating units, as opposed to 1015 transmission connected controllable generation units, in a positive step towards the decarbonisation of energy use [1], [2]. This rapidly expanding integration of devices within the grid, in addition to the increasing susceptibility of the grid to disturbances, is already introducing significant challenges and complexity, compromising the capability of existing monitoring, control, and protection methods [3]. Novel solutions to address these challenges are continuously proposed by the research community and their feasibility often proven only by offline simulations. There is however an increasing need to validate novel solutions in a more realistic environment and at scale, i.e. to prove their scalability and resilience. Offline desktop based simulation tools, such as MATLAB Simulink or PSCAD, offer the capability to undertake detailed modelling of power networks while real-time simulations, constrained by hardware, are typically limited to reduced models or only a small section of the power network. To overcome these limitations, and building upon the real-time simulation resources at independent geographically separated research infrastructures (RIs), the concept of geographically distributed simulations (GDS) was explored.

The term GDS within the power systems context began to appear in 2006 [4] where the conceptual idea of undertaking simulations at more than one geographical location was presented, with first truly geographically distributed implementations reported in 2009 [5], [6]. Two digital real-time simulators (DRTS), one at University of Alberta (Canada) and another at Florida State University (USA) - a distance of $\sim 3,703 \mathrm{~km}$, were coupled to demonstrate geographically distributed simulation for shipboard application. The coupling at this stage was limited to North America, and the early experience did not enforce confidence within the community for its widespread adoption and utilization. With the advancements in communications technologies supported by advanced interfaces, the concept was re-investigated by RWTH Aachen University (Germany) in collaboration with University of South Carolina (USA) in 2015 [7], the first transatlantic implementation across a distance of $\sim 7,359 \mathrm{~km}$. This was soon complemented with a first European implementation, with an interconnection between RWTH and SINTEF (Norway) [8]. A transpacific implementation between National Renewable Energy Laboratory (NREL, USA) and CSIRO (Australia) across a distance of $\sim 13,570 \mathrm{~km}$, the longest interconnection thus far, was reported in [9]. To this point, the implementations were limited between two RIs, whereas the first large scale demonstration referred to as "Global Real-time SuperLab" was reported in [10], a transatlantic interconnection of eight RIs.

The successful implementation and demonstration of GDS and the realization of the growing complexity of power systems, triggered a new wave of interest within the field and is currently a topic of active research. This paper presents the outputs of the IEEE PES Task Force on Interfacing Techniques for Simulation Tools in order to better understand and consolidate the existing developments in GDS in a concrete step towards standardization of the concept. The key contributions of this paper are as follows:

- This paper proposes a generalized taxonomy for potential real time setups that can be utilized within the GDS 
realm. This will ensure consistency in any future development of the concept.

- A thorough appraisal of recent advancements in realtime coupling of geographically separated RIs for GDS is presented. The evaluation encompasses a characterization based on the presented taxonomy, and technological design considerations that underpin the concept.

- Four selected case studies highlighting the practicality of the approach are discussed providing insights into potential wider applications for which the concept can be adopted.

- Furthermore, topics that warrant future research attention have been identified.

The remainder of the paper is organized as follows: the taxonomy of the setups utilized for GDS is presented in Section II, followed by a comprehensive overview in Section III. In Section IV, four selected implementations of GDS covering the breadth of technological design variations are presented, each utilized for applications within smart grid context. Section V presents an outlook, identifying areas that need to be addressed moving forward and concludes the paper.

\section{TAXONOMY OF REAL-TIME Simulations}

The functional distinction between an offline simulation and a real-time simulation is the relation between the simulation time and the elapsed wall-clock time. For an offline simulation, the simulation time can be less than, greater than or equal to the elapsed wall-clock time, while for a real-time simulation the simulation time is guaranteed to be equal to the elapsed wall-clock time. There is more than one approach utilized for power system validation within the real-time simulation realm and this section establishes their taxonomy.

\section{A. Monolithic Simulations}

In this paper, the term monolithic refers to simulations/experiments undertaken in one research infrastructure. The different real-time monolithic simulation approaches as presented in Fig. 1 are described below.

1) Real-Time Simulation (RTS): When the power system and all its associated controls are simulated within the DRTS, the approach is referred to as RTS.

2) Controller Hardware in the Loop (CHIL): When the power system is simulated in real-time within the DRTS while the complete or a proportion of its controls is embedded within an external micro-controller interconnected with the DRTS, the approach is referred to as CHIL.

3) Power Hardware in the Loop (PHIL): When a portion of the power system is simulated in real-time within the DRTS while the remainder of the power system is represented by physical power equipment interconnected to the DRTS, the configuration is referred to as PHIL.

\section{B. Geographically Distributed Simulations}

This section establishes the taxonomy for the configurations utilized under the GDS regime, where more than one RI is interconnected over the Internet. To ensure consistency with the monolithic approaches, three approaches are defined as shown in Fig. 1 and described below.

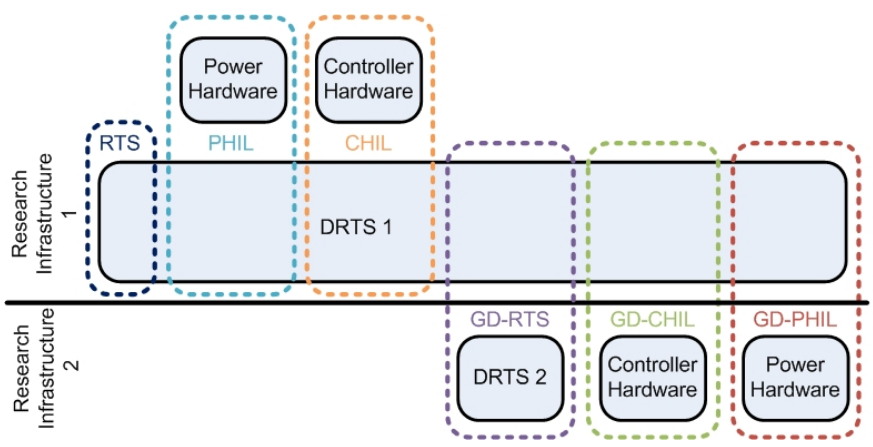

Fig. 1: Classification of monolithic and geographically distributed real-time simulations.

TABLE I: Taxonomical overview of GDS implementations

\begin{tabular}{lc}
\hline Taxonomy & References \\
\hline GD-RTS & {$[5]-[8],[11]-[22]$} \\
GD-CHIL & {$[2]|-26|-[2]$} \\
GD-PHIL & $[9],[10], \mid 21]-[29]$ \\
\hline
\end{tabular}

1) Geographically Distributed Real-Time Simulation (GDRTS): When two DRTS, one at each RI, are interconnected over the internet, the connection is referred to as GD-RTS. Local HIL (PHIL or CHIL) can be incorporated simultaneously at any of the RIs, however, no other geographically distributed interconnection exists.

2) Geographically Distributed Controller Hardware-in-theLoop (GD-CHIL): When the power system is simulated in real-time within the DRTS at one RI while the complete or a proportion of its controls is embedded within an external micro-controller at the second RI and the DRTS and microcontroller are interconnected over the Internet, the approach is referred to as GD-CHIL.

3) Geographically Distributed Power Hardware-in-theLoop (GD-PHIL): When a portion of the power system is simulated in real-time within the DRTS at one RI while the remainder of the power system is represented by physical power equipment at second RI and the DRTS and physical power equipment are interconnected over the Internet, the approach is referred to as GD-PHIL.

It should be noted that an experimental setup can incorporate one or a combination of monolithic and geographically distributed approaches defined by the above taxonomy.

\section{Taxonomical Overview}

A classification of GDS implementations reported in literature, based on the taxonomy proposed in Section II-B, is presented in Table I The first implementations were dominated by GD-RTS, and with further technological advancements these were extended to incorporate controller and power hardware.

\section{TEChNOLOGICAL OVERVIEW}

This section presents an overview of developments from a technological perspective, covering the coupling, the interface and the communications solutions enabling GDS implementations. 


\section{A. Coupling}

There are two types of couplings utilized for GDS within the power systems domain as discussed below:

1) Control Signals Coupling: When control signals are exchanged between the RIs, the coupling is referred to as control signals coupling. This type of coupling was referred to as signal coupling in [4], and examples of such coupling are reported in [23]-[26]. When two different domains are interconnected, such as the thermoelectric coupling presented in [5], [6], no natural coupling exists and the coupling is only a control signals coupling.

2) Electrical Signals Coupling: When electrical signals are exchanged between RIs, the coupling is referred to as electrical signals coupling. Electrical signals coupling is a subset of the natural coupling as identified in [4], where natural coupling refers to coupling of physical systems where conservation of energy is to be maintained. There are three types of electrical signals couplings, asynchronous and synchronous coupling for $\mathrm{AC}$ (i.e., when a power system is split across an AC line) and direct coupling (i.e., when the power system is split across a DC line) as expanded below:

a) Asynchronous AC Coupling: When the two power subsystems, split across an AC line for simulation at two RIs are not synchronized with respect to time, i.e., the voltage and current phase angles at the point of common coupling (PCC) of the two power subsystems are not phase aligned, the coupling is referred to as asynchronous AC coupling. Such a coupling in literature is sometimes referred to as soft real-time. Examples of asynchronous coupling for GDS can be found in [9], [10], [18], [27]-[29].

b) Synchronous AC Coupling: When the two power subsystems, split across an AC line for simulation at two RIs, are synchronized with respect to time, i.e., the voltage and current phase angles at the PCC of the two power subsystems are phase aligned, the coupling is referred to as synchronous AC coupling. The examples of such coupling can be found in [7], [8], [11]-[14], [16], [17], [19]-[21].

c) DC Coupling: When the power system is split across a DC line into two subsystems for simulation at two RIs, the coupling is referred to as DC coupling. Examples in literature for DC coupling are reported in [10], [15].

Remark: The use of control or electrical signals coupling is application driven, where control signals coupling is used if only control signals are exchanged, while electrical signals coupling is used if only electrical signals are exchanged or both if control and electrical signals are exchanged. Similarly, the use of AC coupling or DC coupling is dictated by the system under investigation. However, there does exist a choice between the synchronous and asynchronous AC coupling. Asynchronous coupling was developed as an alternative to synchronous coupling where stringent requirements for hard real-time synchronizations could not be met either due to the delays in communications or the limitations in equipment at RIs that do not support high rate of data exchange and/or update. This lack of hard synchronization therefore limits the applications of asynchronous couplings to steady-state evaluations and slower dynamics studies as has been highlighted in [27]. On the other hand, synchronous AC couplings are capable of transients and dynamics reproduction, limited by the communications delay, i.e., transients and dynamics shorter than the delay itself cannot be accurately reproduced. This therefore implies that synchronous AC coupling can be utilized for transient and fast dynamics studies but also for slower dynamics and steady-state studies and are therefore preferred.

\section{B. Interface}

The coupling with electrical signals within GDS requires selection of an appropriate interface, encompassing an interface algorithm and interface signals, similar to those deployed in a monolithic PHIL setup.

1) Interface Algorithm: The interface algorithm (IA) defines the interconnection setup of the two subsystems of the GDS. The choice of IA impacts the stability of a GDS setup. A number of IAs have been described in the literature for monolithic PHIL setups, as summarized in [30], and can be readily utilized for GDS setups. In early literature for GDS, an IA utilizing a voltage-current overlap decoupling pattern based on relaxation algorithm was proposed in [31]. The use of this interface for coupling terrestrial and shipboard power systems was demonstrated in [4], [32], [33]. An improvement to the IA with capability to handle missing data with numerical approaches such as an extrapolation method was proposed in [12]. However, the IA presented challenges when the system was tightly coupled [34]. An alternative IA was proposed in [34], where a controlled voltage and controlled current source were utilized at either end but required additional resistors for stability. A simpler IA for monolithic PHIL, referred to as the ideal transformer method (ITM), was proposed in [35], requiring only either a controlled current source or a controlled voltage source at each end. The ITM IA established its dominance in GDS due to its straightforward implementation and good stability performance. Examples of its use for GDS $\mathrm{AC}$ coupling (synchronous and asynchronous) can be found in [7]-[10], [13], [14], [16]-[21], [27]-[29]. In [15], the use of ITM IA for DC coupling over an HVDC link was presented with recommendations to ensure stability of the system.

2) Interface Signals and their Transformation: The interface signals for GDS are dictated by the choice of IA utilized. The IAs utilized in [31], [34] require the exchange of measured voltage and current signals from both ends while only one measurement is exchanged from either end when utilizing the ITM IA [35]. This subsection discusses the interface signals and their transformations for the three electrical signals coupling options identified.

a) Synchronous $A C$ Coupling: Early implementation reported in [12] exchanged instantaneous sampled values of a very low frequency $\mathrm{AC}$ signal. The exchange of time domain AC signals (with expected electrical waveform frequency of $50-60 \mathrm{~Hz}$ ) in presence of time delay was proven to be inappropriate for GDS in [19], and the signals are therefore transformed to DC quantities before their exchange. In [20], the interface signals (three-phase waveforms) are decomposed into root mean square (RMS), frequency and phase angle before their exchange while the use of phasor decomposition for GDS was first proposed in [7] and demonstrated in [8]. A detailed analysis of the phasor decomposition for GDS in comparison to monolithic electromagnetic transient simulations was presented in [11]. In [14], a comparison 
of phasor and RMS approaches was presented where higher simulation fidelity under dynamics and transients for phasor based representation was demonstrated and subsequently utilized in [7], [13], [15], [16]. To enhance the stability of GDS setups in the presence of delays, the transformation of threephase waveforms to wave variables (using the analogy of current with velocity and voltage with force) was proposed in [17]. Although the use of wave variables ensured stability for the setup with larger delays, the fidelity of the simulations deteriorated. By supplementing the wave variables by their representation as phasor coefficients, comparable fidelity with improvement in stability was achieved, however, this increases the computational effort. Two shortcomings with use of phasors for GDS were identified: (i) slow speed of response due to its windowing characteristics that can limit reproduction of faster transients (such as phase shifts) [8] and (ii) high computational complexity [21]. To address the challenge of increased computational complexity on resource constrained DRTS, use of an external FPGA node exclusively for phasor decomposition and exchange was discussed in [36]. However, this leads to additional costs (for the FPGA node) and potentially increased delays due to additional interface requirements (between the DRTS and the FPGA node). As an alternative, a custom phasor decomposition model using moving average filter for DRTS was presented in [21]. Citing the high computational complexity of discrete Fourier transform for phasor decomposition and the unavailability of more computationally efficient phasor transformation blocks within DRTS, the use of synchronous reference frame for signal transformation was proposed in [37]. A detailed applicationagnostic comparison with conventional phasor decomposition available within DRTS was presented to establish superior dynamic performance. The use of the approach for frequency control within a transmission network in GD-RTS setup was further discussed and is also presented in more detail in Section V-A. Although a promising approach, the limitation of its use for balanced operating conditions was highlighted.

b) Asynchronous AC Coupling: The use of asynchronous coupling for GDS has only been reported with ITM IA [9], [10], [18], [27]-[29]. In all the reported implementations, the measured voltage from current source end is exchanged as RMS value. In [27], the measured currents from the voltage source end were exchanged as phasors and later also adopted in [9]. In [18], the exchange of active and reactive power measurements from the voltage source end was proposed and later adopted in [10], [28], [29]. The feedback currents are derived from the active and reactive power with the voltage at the current source end as the reference. The exchange of active and reactive powers as opposed to current phasors presented the advantage of a reduced number of interface signals to be exchanged (two - active and reactive power, instead of six - magnitude and phase of three phase currents) while also ensuring conservation of energy at the interface. No other transformation or manipulation of interface signals has been reported.

c) DC Coupling: For DC couplings, the choice of interface signals is also dictated by the choice of IA, however, no transformation is required as the signals to be exchanged are DC quantities [15].
3) Summary of Current Practices in GDS Interface: With evidence of all GDS adopting the ITM IA, it can be concluded that this is the preferred implementation and has been sufficiently derisked for adoption with confidence in future GDS implementations, irrespective of the coupling considered.

For synchronous AC coupling, there are two contenders for signal transformations - the synchronous reference frame and the phasor decomposition. With the use of synchronous reference frame limited to balanced operating conditions, the choice of phasor transformation for unbalanced systems is straightforward, yet the shortcomings of slow response and computational complexity remain. The use of single phase synchronous reference frame has been identified as a potential solution to extend its use to unbalanced systems. For all other cases, there is a lack of a comprehensive comparison of the approaches to derive a definitive conclusion. For Asynchronous AC coupling, the exchange of RMS value of voltage from the current source end and the corresponding active and reactive powers from the voltage source end represents the current practice in literature with advantages of reduced number of interface signals exchange as identified earlier. For DC coupling, the signals are exchanged without the need of any transformation.

\section{Communications}

An overview of communications for GDS is presented for each of the following integral elements.

1) Network: The exchange of interface signals between the RIs for GDS is over the Internet. The latencies involved are dependent upon the technology or the combination of technologies that interconnect the RIs (wired or wireless). The maximum latency between European RIs can be approximated to $\sim 50 \mathrm{~ms}$ (measured as round trip time), which is given by the physical limits of light propagation in optical fiber networks and the geographical distances between the sites. The interconnectivity is provided through GÉANT - the pan-European data network for the research and education community [38]. GÉANT interconnects 19 European countries with data link speeds in magnitude of multiples of $100 \mathrm{Gbps}$, extended Europe with link speeds in magnitude of multiples of $10 \mathrm{Gbps}$, and wider continental Europe with links of 1-9 Gbps. A topology map can be found in [39]. A similar network exists within the USA, referred to as the Energy Sciences Network (ESnet) supported by the Department of Energy [40] and is connected to GÉANT via the transatlantic fibre link between the continents [41].

2) Protocol: Within the first reported distributed implementation [5], [6], an additional external micro-controller was used with the DRTS to facilitate communication with an orchestrator. The micro-controller sent the data to a local server (orchestrator) using Modbus-TCP (Modbus protocol with TCP interface), and the server used UDP to send the data over the Internet. With the development of network interface cards for DRTS, the option of using a number of protocols was made available and the requirement for external micro-controllers was made obsolete [12]. UDP was increasingly preferred over TCP due to its suitability for real-time implementation. Compared to TCP, a connection-oriented protocol, UDP is 
a connection-less protocol that: (i) does not wait for data receipt acknowledgement, thereby reducing overall latency and (ii) does not resend the data in case of data loss, ensuring only the most recent data is updated [27]. More recent GDS implementations dominantly utilized UDP as the protocol as in [7], [8], [10], [13]-[23], [25], [28], [29], [42].

3) Transmission Rate: Transmission rate refers to the rate at which the interface signals are exchanged between the RIs. The transmission rate is either equal to or lower than the simulation time step utilized, limited by the capabilities of the network interface card of the DRTS.

a) Synchronous AC Coupling: For synchronously coupled and DC coupled GDS, time steps in the range of 50$200 \mu \mathrm{s}$ are reported in literature [7], [14], [15], [19], [21], [23], corresponding to a sampling rate in the range of 5$20 \mathrm{kHz}$, however the maximum supported transmission rate of network interface card reported is $10 \mathrm{kHz}$ [37]. Typically, a static transmission rate is utilized where the sending rate and the receiving rate is same. When data is exchanged using UDP, the receiving rate can be lower than the sending rate due to loss of packets (and no retransmission) as is the recognized characteristic of the protocol. An analysis of the impact of different transmission rates for phasor representation of interface signals was presented in [19], where two findings were reported: (i) the latency increased with increase in transmission rate and (ii) deterioration in accuracy of the signal reproduction when transmission rate was decreased. This therefore presented a trade-off and a $2 \mathrm{kHz}$ transmission rate for reproduction of electrical signal (sine wave) was found appropriate given the latency exhibited. In [14], a comparison between RMS and phasor representation with varying transmission rates was presented. In both cases, deterioration in accuracy with decrease in transmission rate was reported, with a recommendation of $2 \mathrm{kHz}$ for appropriate signal reproduction. In [21], the use of real-time control protocol (RTCP) for adaptive transmission rate was reported. Although UDP was utilized as the underlying transport protocol, RTCP allowed for data transmission rate manipulation based on quality of service. An additive increase multiplicative decrease (AIMD) scheme which linearly increases the transmission rate in the case of no congestion and reduces the transmission rate by a multiplicative factor in case of a congestion [43].

b) Asynchronous AC Coupling: A wide range of time steps were reported for asynchronous coupling, ranging from $50 \mu \mathrm{s}$ to $2 \mathrm{~s}$ [9], [10], [27]-[29], and in all cases the transmission rate was less than the corresponding sampling rate $(0.5 \mathrm{~Hz}$ in [9], $1 \mathrm{~Hz}$ in [27] and $100 \mathrm{~Hz}$ in [28], [29] and $1 \mathrm{kHz}$ in [10].

c) DC Coupling: As mentioned earlier, the feasibility of DC coupling was proven in [15] via a local system decoupling with incorporation of static delay to emulate geographical separation. The transmission rate therefore utilized was $20 \mathrm{kHz}$, equal to the corresponding simulation time step of $50 \mu \mathrm{s}$. In the truly distributed implementation of DC coupling reported in [10], a transmission rate of $2 \mathrm{kHz}$ was shown to be appropriate for the application.

4) Orchestrator: An orchestrator, also referred to as coordinator, gateway or broker, is an entity that facilitates communication between the interconnected RIs. This can involve (i) the accommodation of different protocols, data formats and respective conversions to allow vendor agnostic interconnection of experimental equipment [5], [6], [8]-[13], [16], [18], [19], [21]-[23], [44], (ii) synchronization of data if required [9], [27], (iii) to bypass local firewalls [25], [29], and (iv) data logging and visualization [9], [10]. An orchestrator can be implemented locally within one of the RIs [18], [23], locally within both RIs [5], [6], [12], [16], [21], [22], [25], [27], [28], can be hosted in the cloud [9], or a combination of approaches [8], [10], [11], [13], [19], [29], [44].

Most works in literature report a custom implementation, a script in Microsoft Visual C++ [5], [6], a script in python [23], [25], [27], or utilize functionalities of existing commercial packages such as LabVIEW in [12]. Most of the implementations rely on existing libraries/data notations for enabling the required functionalities, and the use of JavaScript Object Notation [27], Google's Protocol Buffers [9] and Remote Procedure Calls [25] has been reported. A platform that facilitated orchestration of experiments across multiple RIs was presented in [9]. This platform was designed for asynchronous couplings and was not made available openly. In [26], an open source orchestrator referred to as Joint Test Facility for Smart Energy Networks with Distributed Energy Resources (JaNDER), was proposed. The incorporation of the orchestrator although facilitated the interconnection of RIs, the delays introduced were in order of seconds and thereby limiting its application to asynchronous AC couplings for steady-state and slower dynamics studies [29]. In [13], the requirements for a framework that would support synchronous AC couplings were reported. Building upon the requirements an open source framework, referred to as virtually interconnected laboratories for large systems simulation (VILLAS), was first presented in [19] and offered a comprehensive suite of services required of an orchestrator (such as data conversion, visualisation, and synchronization). The framework was adopted in [10], [11], [16], [18], [21], [28] and more recently further improvements to enhance the interoperability of the framework was reported in [44]. An FPGA compatible version to support synchronous AC couplings was proposed in [36].

5) Cyber-Security: GDS encompasses exchange of information across multiple RIs over the Internet. Secure transmission of information is crucial due to two reasons: (i) the information being exchanged or the experiments being undertaken can be sensitive in nature (commercially or otherwise) and (ii) this information can be driving hardware equipment that can be susceptible to false data injection as in [45], [46]. Although a minimum/maximum range protection at each participating equipment is locally implemented within each RI, manipulation of data can still risk abnormal behaviour. In a few implementations, the use of virtual private network (VPN) is reported to ensure secure transmission of data between the RIs [7], [8], [10], [13], [16], [18], [21], [22], [27], [28], [42]. A slight increase in latency due to the use of VPN has been reported in [19]. Some other implementations, such as [9], [25], [26], [29], rely on the encryption offered by the underlying software libraries utilised.

6) Time Delay Determination and Compensation: The exchange of interface signals between the two subsystems introduces delay. In monolithic PHIL setups, the delay is variable yet deterministic [47]. However, for GDS setups where communication is over the Internet, the delay is non- 
deterministic and time varying. Accurate determination of the delay requires a time synchronized clock signal at each end. Time synchronization can be achieved, either using public network time protocol (NTP) over the Internet or the global positioning system (GPS) NTP. A public NTP is economical but relies on frequent pinging for synchronization; no reported implementations utilize a public NTP.

a) Synchronous AC Coupling: For reliable operation, such as that required for synchronous coupling, GPS time synchronization becomes imperative, requiring a GPS clock at either end of the GDS setup [13], [16], [20], [21], [37]. Time delay within monolithic PHIL setups can be compensated using a lead filter [48] or a linear predictor [20]. Accuracy of compensation methods such as the lead filter and linear predictor is highly dependent upon the setup, their design and they do not perform well under time varying delays. An alternative approach, where phase of the electrical signals are shifted by an amount equivalent to the time delay, was proposed in [49]. The approach is independent of the setup and performs well under varying time delays (obtained using GPS) and therefore has been adopted for GDS in [10], [13], [14], [17], [19], [44].

b) Asynchronous AC Coupling: The need for time delay compensation in asynchronous coupling is sometimes eliminated by the use of time steps much larger than the communication delay itself, for example as in [9], [27]. The use of simple estimator based compensation was proposed in [28], however the method does not ensure conversation of energy at the point of coupling. In cases where slower dynamics are of interest, use of feed-forward control compensation to time align control and power commands at multiple RIs was proposed as a potential solution and identified as future work in [29].

c) DC Coupling: Time delay compensation techniques for DC couplings have not been reported in literature, partly due to the fact that the steady state accuracy is not impacted as in the case of AC couplings. The reported DC coupling for GDS in [10], [15] does not incorporate any time delay compensation.

7) Summary of Current Practices in Communications for GDS: The Internet is identified as the key enabler for GDS. Although a choice of high bandwidth connections are available within each country, the network that interconnects the RIs is not under the control of participating RIs but rather dictated by the existing infrastructure. In terms of the protocol, UDP offers the desired characteristics for stable GDS with minimal latency. With varied examples in literature, a transmission rate of $2 \mathrm{kHz}$ or higher for synchronous and DC couplings and $1 \mathrm{kHz}$ or lower for asynchronous coupling is recommended. VILLAS as orchestrator satisfies all the requirements for GDS while JaNDER can be chosen in asynchronous couplings due to its light-weight implementation. Both, VILLAS and JaNDER, offer data encryption before exchange satisfying the security concerns. The phasor based time delay compensation offers the most accurate compensation for synchronous AC couplings while more research to improve and accommodate delays in asynchronous couplings is required.

\section{Brief summary of contributions}

In Table [I] the works in literature that have made a contribution to the development and advancement of GDS have been summarized.

TABLE II: Summary of contributions to GDS development

\begin{tabular}{|c|c|c|}
\hline Contribution to & & Description \\
\hline \multicolumn{3}{|r|}{ Couplings } \\
\hline $\begin{array}{l}\text { Control Signals } \\
\text { Coupling }\end{array}$ & $|30|$ & $\begin{array}{l}\text { First reported GDS control signals coupling, } \\
\text { thermo-electric simulation coupling of two } \\
\text { domains }\end{array}$ \\
\hline $\begin{array}{l}\text { Electrical Signals } \\
\text { Coupling }\end{array}$ & $\frac{81}{27]}$ & $\begin{array}{l}\text { First reported synchronous AC coupling } \\
\text { First reported asynchronous AC coupling }\end{array}$ \\
\hline \multicolumn{3}{|r|}{ Interface } \\
\hline $\begin{array}{l}\text { Interface } \\
\text { Algorithm }\end{array}$ & \begin{tabular}{|l|}
30 \\
31 \\
12 \\
34 \\
15 \\
15 \\
15 \\
\end{tabular} & $\begin{array}{l}\text { A review and comparison of IAs } \\
\text { Voltage-current decoupling pattern IA } \\
\text { Extension of IA proposed in [30] with ca- } \\
\text { pability to handle missing data } \\
\text { Generalized coupling scheme IA } \\
\text { ITM IA } \\
\text { Use of ITM IA for DC coupling }\end{array}$ \\
\hline $\begin{array}{l}\text { Interface } \\
\text { Signals }\end{array}$ & 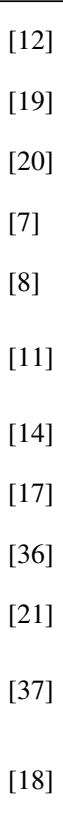 & $\begin{array}{l}\text { Instantaneous values of interface signals for } \\
\text { exchange between RIs } \\
\text { Analysis of instantaneous time domain val- } \\
\text { ues for GDS } \\
\text { RMS values of interface signals for ex- } \\
\text { change between RIs } \\
\text { Phasors of interface signals for exchange } \\
\text { between RIs } \\
\text { Feasibility of Phasors as interface signals } \\
\text { for GDS } \\
\text { Analysis of phasor decomposition with } \\
\text { monolithic electromagnetic transient simu- } \\
\text { lation } \\
\text { A comparison of phasor vs RMS for inter- } \\
\text { face signal exchange } \\
\text { Representation of interface signals as wave } \\
\text { variables for exchange between RIs } \\
\text { FPGA external to DRTS for phasor decom- } \\
\text { position. } \\
\text { Phasor decomposition using moving aver- } \\
\text { age filter } \\
\text { Synchronous reference frame transforma- } \\
\text { tion of interface signals for exchange be- } \\
\text { tween the RIs } \\
\text { Use of active and reactive power values for } \\
\text { exchange between RIs instead of current } \\
\text { phasors for asynchronous coupling }\end{array}$ \\
\hline \multicolumn{3}{|r|}{ Communications } \\
\hline $\begin{array}{l}\text { Transmission } \\
\text { Rate }\end{array}$ & [19] & $\begin{array}{l}\text { Analysis of impact of transmission rate on } \\
\text { latency } \\
\text { A scheme for adaptive transmission rate } \\
\text { based on network quality of service }\end{array}$ \\
\hline Orchestrator & \begin{tabular}{|l|}
91 \\
26 \\
29 \\
13 \\
194 \\
136 \\
19 \\
\end{tabular} & $\begin{array}{l}\text { Platform that supported orchestration of ex- } \\
\text { periments across multiple RIs } \\
\text { Open source orechestrator platform JaN- } \\
\text { DER } \\
\text { Latency analysis of JaNDER } \\
\text { Requirements of orchestrator platform } \\
\text { Open source orchestrator platform VILLAS } \\
\text { Improvements to VILLAS platform reported } \\
\text { VILLAS platform for FPGA developed }\end{array}$ \\
\hline Cyber-Security & [19] & Analysis of impact of VPN on latency \\
\hline $\begin{array}{l}\text { Time Delay } \\
\text { Compensation }\end{array}$ & \begin{tabular}{|l|}
$\frac{47}{48}$ \\
$\frac{20}{\mid 20}$ \\
$\frac{29}{28}$ \\
\end{tabular} & $\begin{array}{l}\text { Time delay characterization of PHIL } \\
\text { Lead filter compensation } \\
\text { Delay compensation with linear predictor } \\
\text { Phase-shift delay compensation } \\
\text { Estimator-based delay compensation }\end{array}$ \\
\hline
\end{tabular}

*References [6], [10], [16], [22]-[25] present examples of use of one or more of the technologies discussed above. 


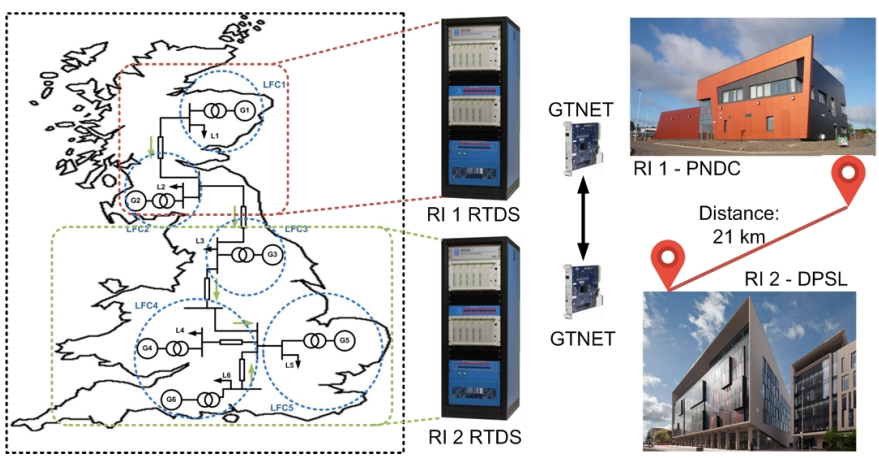

Fig. 2: GD-RTS setup for frequency control of a transmission system [37|.

\section{Case Studies}

In this section, four selected case studies, encompassing different geographical, taxonomical, and technological options are presented.

\section{A. Frequency Control within a Large Transmission System}

A schematic overview of this implementation is shown in Fig. 2 and summarized in Table III. The two RIs are part of the University of Strathclyde (UST) - the Dynamic Power Systems Laboratory (DPSL) and the Power Networks Demonstration Centre (PNDC), separated over a distance of $21 \mathrm{~km}$.

Objective: To determine the suitability of (i) synchronous reference frame transformations for GDS, (ii) splitting a transmission network for GDS and (iii) capturing sub-second dynamics for frequency control studies using GDS.

Implementation: A reduced dynamic model of the Great Britain power system was utilized [50]. In contrast with other cases in the literature, the study proposes a synchronous frame transformation of the interface signal, offering improved dynamics and reduced computational effort in comparison to a conventional phasor transformation but limited to balanced operating conditions.

Results: The study presented an application agnostic and application oriented performance characterization to establish the advantages of the proposed synchronous reference transformation of interface signals. The application agnostic comparison demonstrated improved response with minimal errors under steady state, dynamic and transient events, in reproducing harmonics with selective time delay compensation,

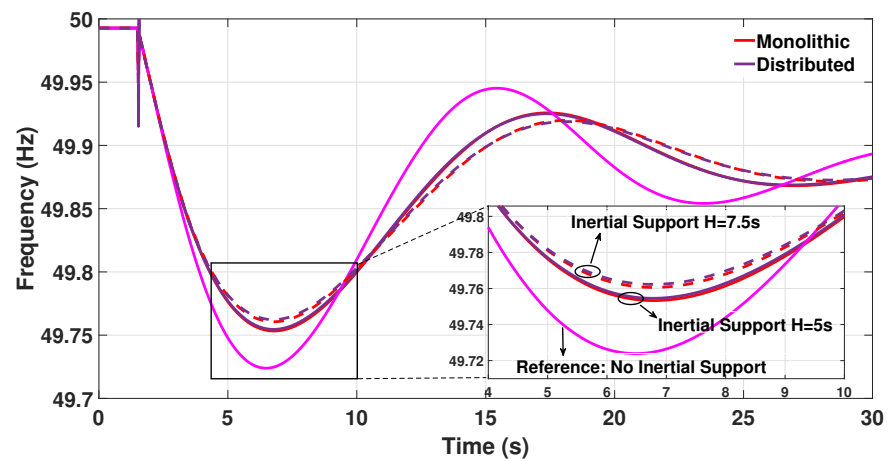

Fig. 3: Inertial response demonstration using GD-RTS [37].

and under variable communications delay. The setup in Fig. 2 served for application oriented performance characterization of the proposed interface where four parameters for frequency control were characterized at the PCC, i.e., frequency, rate of change of frequency, active power and reactive power. Having established the superior performance of the proposed interface and the close proximity to the results compared to a monolithic setup, its use for swing equation based inertial response was demonstrated. A comparison of frequency responses for three scenarios (no inertial support, inertial support with inertia constant $H=5 \mathrm{~s}$ and $H=7.5 \mathrm{~s}$ ) are shown in Fig. 3 With the incorporation of inertial support, i.e., provision of additional active power, the slope and nadir of the response are improved. There is limited improvement when $H$ is increased from 5 to 7.5 as the amount of active power reserve within the network is constrained. As can be observed, the monolithic and distributed results are in close proximity with the error associated to the accuracy of the proposed interface well within the characterized frequency error of $8.744 \times 10^{-5}$.

Insight offered and future considerations: The work has established evidence of the feasibility of large system simulations and subsequently that of capturing sub-second dynamics using GDS. Two directions have been identified by authors for future work: (i) feasibility of single phase synchronous reference frame transformations to overcome the limitation of the interface applicability to balanced operating conditions and (ii) establishing the feasibility of the approach for transient studies (such as protection studies).

TABLE III: Summary of implementation A

\begin{tabular}{|c|c|c|c|c|c|}
\hline \multirow{2}{*}{ Geographical } & Distance & Number of RIs & \multicolumn{3}{|c|}{ RIs Involved } \\
\hline & $21 \mathrm{~km}$ & 2 & \multicolumn{3}{|c|}{ UST: DPSL and PNDC } \\
\hline Taxonomy & \multicolumn{5}{|c|}{ GD-RTS } \\
\hline Coupling & \multicolumn{5}{|c|}{ Electrical Signals Coupling: Synchronous AC Coupling } \\
\hline \multirow{2}{*}{ Interface } & Algorithm & Transformation & \multicolumn{3}{|c|}{ Signals } \\
\hline & ITM & Synchronous Frame & \multicolumn{3}{|c|}{$\begin{array}{l}\text { DPSL to PNDC: Synchronous transform of Voltage, angular frequency and time stamp } \\
\text { PNDC to DPSL: Synchronous transform of Current, angular frequency and time stamp }\end{array}$} \\
\hline \multirow{2}{*}{ Communications } & Protocol & Transmission Rate & Orchestrator & Compensation & Security \\
\hline & UDP & $10 \mathrm{kHz}$ & No Orchestrator & Phase Compensation & No VPN \\
\hline
\end{tabular}




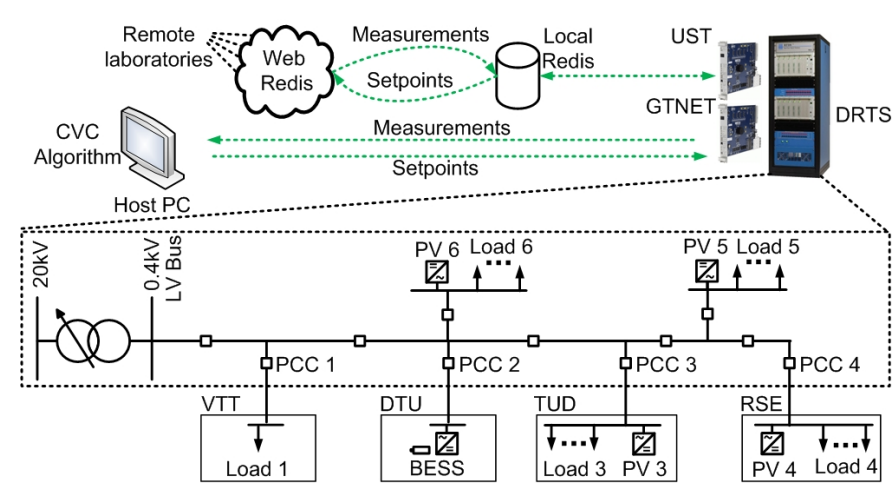

Fig. 4: GD-PHIL setup for voltage control of a distribution network [29].

\section{B. Voltage Control of a Distribution Network}

A schematic overview of the implementation is shown in Fig. 4 and summarized in Table IV] Five RIs were involved in the experiment: UST, Denmark Technological University (DTU, Denmark), Delft University of Technology (TUD, Netherlands), Ricerca sul Sistema Energetico (RSE, Italy) and Technical Research Centre of Finland (VTT). The farthest distance being between UST and VTT at $\sim 1780 \mathrm{~km}$.

Objective: To demonstrate the suitability of asynchronous electrical signals coupling within a GD-PHIL setup for slower dynamic studies such as voltage control.

Implementation: A coordinated voltage control (CVC) [51] is employed within CIGRE low voltage test network with the power network being split across UST and TUD while incorporating hardware (battery energy storage system BESS, photovoltaic PV, and load bank) from the other participating RIs. The CVC is incorporated within a CHIL implementation at UST. Data exchange is facilitated by JaNDER [26] as an orchestrator, with cloud and local implementation referred to as Web Redis and Local Redis respectively. Voltage control is typically assessed over the duration of a day to characterize its efficacy to deal with varying PV outputs due to solar irradiation and varying load profiles. For the purpose of realtime implementation, the simulation is accelerated with $1 \mathrm{~s}$ simulation time representing 1 minute of the day. The CVC is run every 15 minutes, i.e., every $15 \mathrm{~s}$ in simulation.

Results: The preliminary results include voltage profile

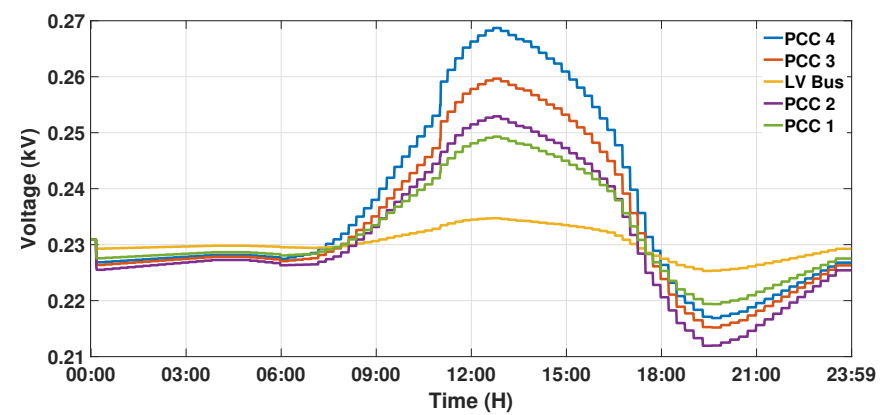

(a) Voltage profiles without CVC.

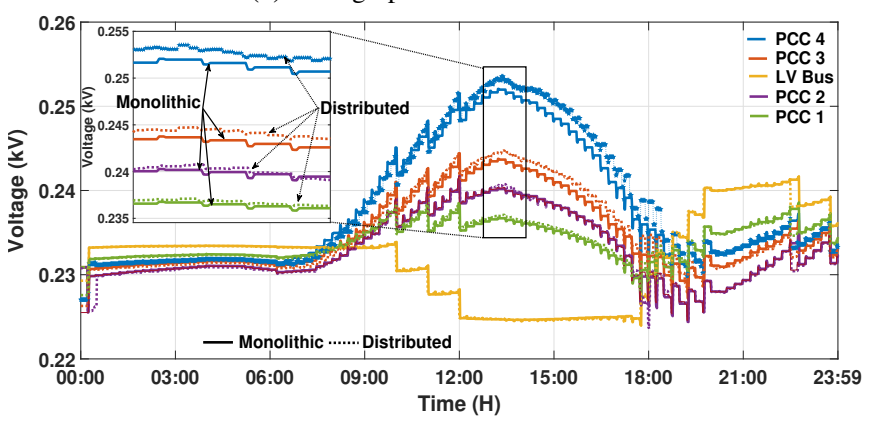

(b) Voltage profiles with CVC.

Fig. 5: Results of CVC using GDS setup |29|

without CVC (Fig. 5a) and with CVC (Fig. 5b) for both monolithic and distributed implementations. The minor differences present in the voltage profiles are associated to the error margin of the approach yet demonstrating the suitability of the asynchronous electrical signals coupling for these slow dynamic studies.

Insight offered and future considerations: The case study establishes evidence of the feasibility of asynchronous coupling for slower dynamic studies over multiple RIs. However, the authors point out the need to characterize the error margin of the approach in comparison to a synchronous approach. The authors highlight that if a relatively close error margin between synchronous and asynchronous coupling for slower dynamic studies is established, the approach would enable incorporation of a larger number of RIs as asynchronous coupling relaxes the requirements in terms of latency, time synchronization and compensation.

TABLE IV: Summary of implementation B

\begin{tabular}{|c|c|c|c|c|c|}
\hline \multirow{2}{*}{ Geographical } & Distance & Number of RIs & \multicolumn{3}{|c|}{ RIs Involved } \\
\hline & $\sim 1780 \mathrm{~km}$ & 5 & \multicolumn{3}{|c|}{ UST, DTU, TUD RSE and VTT } \\
\hline Taxonomy & \multicolumn{5}{|c|}{ GD-PHIL } \\
\hline Coupling & \multicolumn{5}{|c|}{ Electrical Signals Coupling: Synchronous AC Coupling and Control Signals Coupling } \\
\hline \multirow{2}{*}{ Interface } & Algorithm & Transformation & \multicolumn{3}{|c|}{ Signals } \\
\hline & ITM & RMS & $\begin{array}{c}\text { Electrical } \\
\text { Control }\end{array}$ & $\begin{array}{r}\text { UST to Ren } \\
\text { Remote } \\
\text { UST to Remote RI } \\
\text { Remote RIs t }\end{array}$ & $\begin{array}{l}\text { nitude and frequency } \\
\text { id reactive power } \\
\text { power setpoints from CVC } \\
\text { tage magnitude power }\end{array}$ \\
\hline \multirow{2}{*}{ Communications } & Protocol & Transmission Rate & Orchestrator & Compensation & Security \\
\hline & UDP & $100 \mathrm{~Hz}$ & Web - Redis & No Compensation & No VPN \\
\hline
\end{tabular}




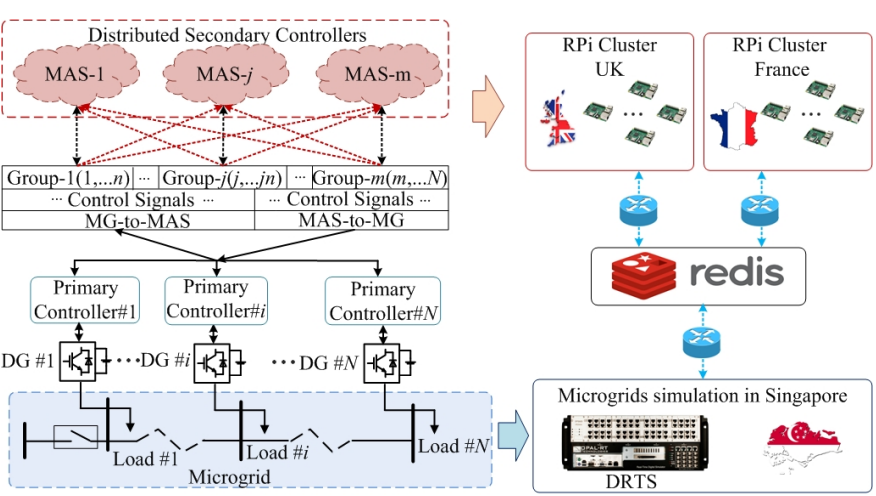

Fig. 6: RT-GD-CHIL setup for frequency and voltage control of microgrids [25]

\section{Frequency and Voltage Control of Microgrids}

A schematic overview of the implementation between three RIs, UST, Nanyang Technological University (NTU, Singapore) and Grenoble Institute of Technology (GINP, France) is shown in Fig. 6 and summarized in Table $\mathrm{V}$. The farthest distance being between UST and NTU at $\sim 11,020 \mathrm{~km}$.

Objective: To demonstrate the premise of operating microgrids remotely, an application within the realm of energy internet.

Implementation: The electrical systems including networks, loads, and DGs with local controls are all emulated in realtime simulator OPAL-RT at NTU. The distributed secondary controllers are implemented in embedded systems remotely at GINP and UST. The remote distributed secondary controllers are exchanging information with electrical systems through cloud server on REDIS. Additionally, the embedded systems also communicate with neighbors via local area networks, which is developed based on the NS3 simulation tool. The communication delay are incorporated by the NS3 tool. In this research, a scalable distributed control approach is designed for microgrids governed by multiple entities. It achieves the secondary control function of microgrids with local and group information exchange. The communication delays are not compensated, rather the stability of the deployed control with delays is proven. In the test case, ten distributed generators (DGs) are controlled from geographically remote RIs within a GD-CHIL setup, five at GINP and five at UST.

Results: The voltage and frequency responses for a step change in load (increase at $t=60 \mathrm{~s}$ and decrease at $t=120 \mathrm{~s}$ )

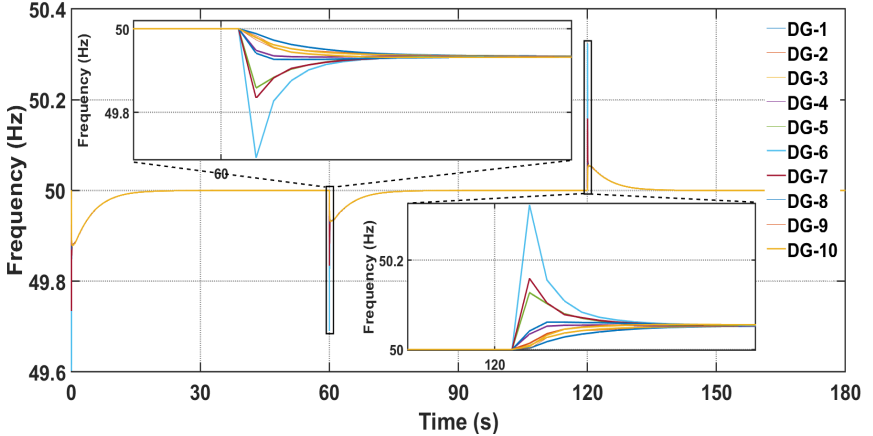

(a) Frequency within microgrid.

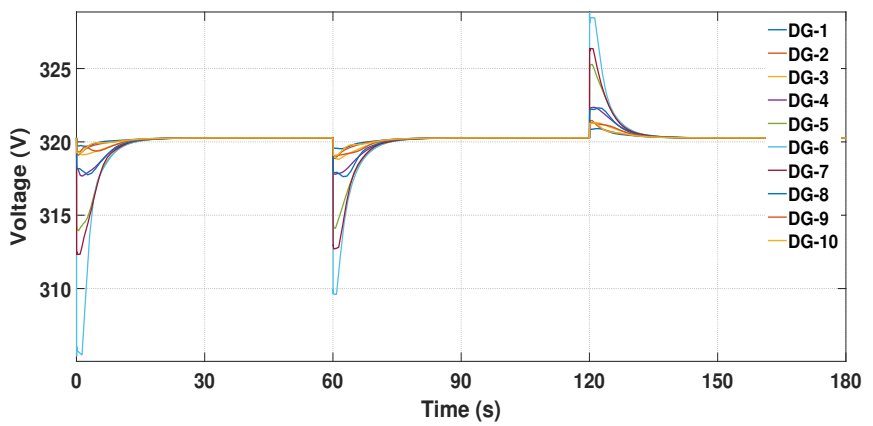

(b) Voltage profiles within microgrid.

Fig. 7: Results of CVC using GDS setup.

for the GD-CHIL implementation is shown in Fig. $7 \mathrm{a}$ and $7 \mathrm{~b}$ respectively. The system frequency and voltage can be restored to nominal values with the proposed control framework. The correct response of the system to the geographically separated controllers indicates the applicability of this solution. More details of the implementation and additional test results can be found in [25].

Insight offered and future considerations: The research primarily establishes evidence of the feasibility of remote control of microgrids governed by multiple entities over Energy Internet. While at the same time, the study provides evidence of the use of GD-CHIL for validation of distributed control algorithms. GD-CHIL setups offer the capability to validate the scalability of distributed control algorithms, an important aspect that limits their real-world adoption. More extensive setups with large number of distributed controllers are the next step in realizing the true potential the approach has to offer.

TABLE V: Summary of implementation C

\begin{tabular}{|c|c|c|c|c|c|}
\hline \multirow{2}{*}{ Geographical } & Distance & Number of RIs & \multicolumn{3}{|c|}{ RIs Involved } \\
\hline & $\sim 11,020 \mathrm{~km}$ & 3 & \multicolumn{3}{|c|}{ UST, NTU, and GINP } \\
\hline Taxonomy & \multicolumn{5}{|c|}{ GD-CHIL } \\
\hline Coupling & \multicolumn{5}{|c|}{ Control Signals Coupling } \\
\hline \multirow{2}{*}{ Interface } & Algorithm & Transformation & \multicolumn{3}{|c|}{ Signals } \\
\hline & - & - & \multicolumn{3}{|c|}{$\begin{array}{l}\text { NTU to UST and GINP: voltage and frequency measurements } \\
\text { UST and GINP to NTU: active and reactive power setpoints }\end{array}$} \\
\hline \multirow{2}{*}{ Communications } & Protocol & Transmission Rate & Orchestrator & Compensation & Security \\
\hline & UDP & $100 \mathrm{~Hz}$ & Web - Redis & No Compensation & No VPN \\
\hline
\end{tabular}




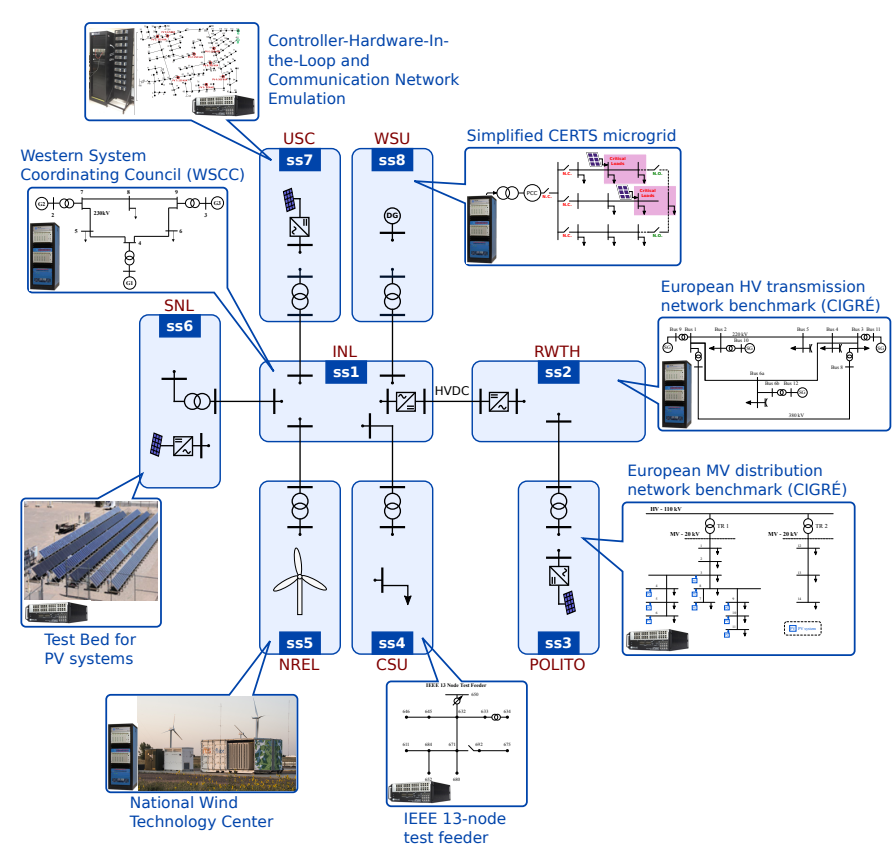

Fig. 8: Global Real-time SuperLab [10].

\section{Transnational HVDC Coupling of Transmission Systems in Europe and the US}

An overview of the Global Real-time SuperLab experiment is shown in Fig. 8 and summarised in Table VI. In 2017, it interconnected a total of ten DRTS across eight geographically distributed simulation sites [10]. The complexity of the setup became manageable with VILLAS framework which exchanged interface signals, centrally collected simulation results and monitored the execution via a web-based interface [44]. RWTH Aachen conducted the simulation of a European CIGRE HV transmission network, while the Idaho National Lab (INL) simulated the Western System Council (WSCC) 9-bus system. Both networks were coupled by a long-distance HVDC link. In addition to GD-RTS, National Renewable Energy Laboratory (NREL) implemented a local PHIL setup with a wind turbine and the University of South Carolina (USC) incorporated a local CHIL setup for photovoltaic inverter control and a communications network emulator. The remaining RIs, Washington State University (WSU), Sandia National Lab (SNL), Colorado State University (CSU) and Polytechnic University of Turin (Polito) contributed

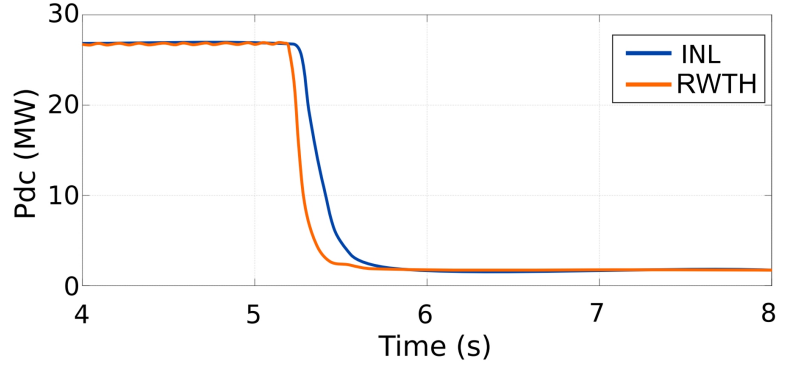

(a) DC Power through HVDC link.

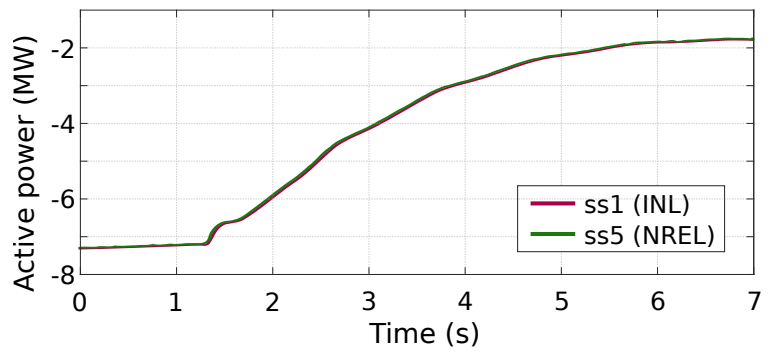

(b) Active power at INL-NREL PCC.

Fig. 9: Results from Global Real-time SuperLab setup.

to the GD-RTS with simulation of several distribution systems (as identified in Fig. 8) interconnected to the CIGRE and WSCC networks. As a central site, INL hosted three real-time simulators from RTDS, OPAL-RT and Typhoon which have been coupled locally with the central 9-bus system.

Objective: To demonstrate the feasibility of long distance and complex GD-RTS scenarios with a heterogeneous selection of DRTS tools with a globally interconnected power grid based on Ultra HVDC lines connecting continents [52].

Implementation: The transatlantic coupling of the $50 / 60 \mathrm{~Hz}$ systems has been established by a HVDC link and an ITM IA [15]. For the transmission - distribution coupling between INL/RWTH and other RIs, an asynchronous coupling based on $V_{r m s}, f, \phi$ and $P, Q$ injections has been used. All DRTS used a timestamp of $50 \mu \mathrm{s}$ and exchanged their interface signals at $1 \mathrm{kHz}$.

Results: Fig. $9 \mathrm{a}$ and $9 \mathrm{~b}$ present the propagation of an event originating in the European CIGRE network following a change of power reference in the HVDC converter station, and the consequent frequency transient for the generators of the WSCC network and for the generator power of the windturbine within the PHIL setup of NREL's distribution system.

TABLE VI: Summary of implementation D

\begin{tabular}{|c|c|c|c|c|c|}
\hline \multirow{2}{*}{ Geographical } & Distance & Number of RIs & \multicolumn{3}{|c|}{ RIs Involved } \\
\hline & $\sim 7955 \mathrm{~km}$ & 8 & \multicolumn{3}{|c|}{ RWTH, INL, NREL, USC, WSU, SNL, CSU, Polito } \\
\hline Taxonomy & \multicolumn{5}{|c|}{ CHIL, PHIL \& GD-RTS } \\
\hline Coupling & \multicolumn{5}{|c|}{ Electrical Signals Coupling: Asynchronous AC \& DC Coupling } \\
\hline \multirow{2}{*}{ Interface } & Algorithm & Transformation & \multicolumn{3}{|c|}{ Signals } \\
\hline & ITM & RMS & \multicolumn{3}{|c|}{$\mathrm{AC}: V_{r m s}, f, \phi+P, Q$ DC: V/I } \\
\hline \multirow{2}{*}{ Communications } & Protocol & Transmission Rate & Orchestrator & Compensation & Security \\
\hline & UDP & $1 \mathrm{kHz}$ & VILLASweb & No Compensation & Tinc-VPN \& IPsec \\
\hline
\end{tabular}


These results prove that GD-RTS is a viable methodology to study the propagation of events across multiple coupling points.

Insight offered and future considerations: The case-study demonstrated the feasibility of a large scale distributed realtime simulation. A major challenge was the management and coordination of the involved participants as the institutions cross state, country and timezone borders. The scheduling of common time-slots was challenging and was relieved by many smaller bi-lateral test sessions before the full system has been simulated as a whole. The careful preparation of pre-validated example model files for the involved simulation platforms was key to the success of the demonstration. Also the leading role of a central site (here INL) simplified the coordination as a central point of contact and monitoring via the web-interface was possible. More GD-RTS complex topologies deviating from the star topology are likely to reveal new challenges.

\section{OUTLOOK}

GDS as a concept has been around for over a decade and with timely renewed interest in the face of emerging need, there are opportunities to capitalize to push the boundary of the concept. This section presents the more immediate research and development opportunities as identified by the PES Task Force on Interfacing Techniques for Simulation Tools.

System partitioning and initialization: Performing system partitioning appropriately can improve system stability, and enhance simulation performance and accuracy. With the growing size of the test system, appropriate system partitioning will move from a desired feature to a requirement. Furthermore, the complexity of distributed power system models is expected to increase with the objective of realising closer to real-world representation of increasing complex smart grids. Such a setup may involve several diverse tools and tool-chains, requiring working with different editors and languages, running on different platforms. Initializing such a setup in a synchronized manner presents significant challenges where all participating tools need to have coordinated initialization values when starting the simulation. Efforts to realize such an approach for GDS are necessary.

Latency: A well known but always relevant aspect is network quality of service. The Internet infrastructure continues to grow in bandwidth and throughput in the past and new dedicated services with low latency for wider applications can be expected in the future. If real-time capable channels with sufficiently low latency become economically available, the use of dedicated instrumentation protocols that are normally only in use for local connections (e.g. the Aurora protocol) may be readily utilized. The same holds true for time synchronization methods where economical alternatives are sought. Until then, it will be important to have clarity on the price we pay (in terms of accuracy and fidelity) for "sloppy" coupling, trying to get along with degraded network performance [53].

Simultaneously, novel time-delay compensation techniques that can improve the fidelity of GDS with the limited bandwidth available should be explored. A careful characterization of latency within a setup, as presented in [47] for monolithic setups, can be extended to GDS. This can form a basis for innovative compensation techniques such as probabilistic compensation or data-driven compensation to ensure higher fidelity and accuracy.

Cyber-security: Limited attention has been paid to secure transmission of information, with approaches relying on a virtual private network reported in literature. Incorporating additional security measures can impact the speed of data transmission while might be necessary for protection of hardware when multiple RIs are integrated within an experiment and therefore requires a careful consideration moving forward.

Interoperability: GDS will involve working with potentially a large number of equipment and applications at every RI, where their integration often presents a challenge. Interoperability is increasingly desired to facilitate seamless integration. A recent development, the Distributed Co-Simulation Protocol (DCP, [54]) by the Modelica Association, addresses the issue at the applications end of a co-simulation setup - greatly improving interoperability within heterogeneous offline simulation setups. Similar advances are required within the realm of GDS.

\section{CONCLUSIONS}

There is a growing need for flexible systems level studies to be undertaken given the transformation the energy system is undergoing: increasing penetration of distributed energy resources coupled via power electronic interfaces, the transition of distribution network operators to distribution system operators, growing numbers of smart grid participants, and the tighter integration of multiple energy vectors to name a few. Not only the computational capabilities have to grow with the problem, but also the diversity of laboratory equipment (such as real machines and controllers), the ability to dynamically integrate with heat and transport systems, and especially the required expertise of scientists. Teaming up across multiple geographically separated research institutes is one of the effective approaches identified. This paper presented the experiences of the IEEE PES Task Force on Interfacing Techniques for Simulation Tools. Combining geographically separated research infrastructures opens up new and significant value, since complementing capabilities and expertise increases the potential coverage of experimental analysis. The reported examples present and discuss experiences from the novel use of coupled research platforms to collaborate on the validation of emerging power systems concepts by sharing equipment, models, computational power and expertise. Still, the here identified constraints remain relevant: latency, optimal interfacing (system partitioning and initialization), and interoperability. These are important and will only be addressed by continued and escalated international cooperation to push the boundaries of real-time geographically distributed simulations.

\section{REFERENCES}

[1] IET. Electricity networks - handling a shock to the system. [Online] Available: https://www.theiet.org/media/2785/elec-shock-tech.pdf

[2] Energy UK. Pathways for the GB Electricity Sector to 2030. [Online]. Available: https://www.energy-uk.org.uk/publication.html? task=file.download\&id=5722

[3] Ofgem. Technical report on the events of 9 august 2019. [Online]. Available: https://www.ofgem.gov.uk/system/files/docs/2019/ 09/eso_technical_report_-_final.pdf

[4] H. P. Figueroa et al., "A modular real-time simulation platform based on the virtual test bed," in 2006 IEEE International Symposium on Ind. Electron., vol. 2, July 2006, pp. 1537-1541. 
[5] M. O. Faruque, et al., "Geographically distributed thermo-electric cosimulation of all-electric ship," in 2009 IEEE ESTS, April 2009, pp. 36-43.

[6] M. O. Faruque et al., "Thermo-electric co-simulation on geographically distributed real-time simulators," in 2009 IEEE Power Energy Society General Meeting, July 2009, pp. 1-7.

[7] M. Stevic et al., "Development of a simulator-to-simulator interface for geographically distributed simulation of power systems in real time," in IECON 2015, Nov 2015, pp. 005 020-005025.

[8] M. Stevic et al., "Feasibility of geographically distributed real-time simulation of hvdc system interconnected with ac networks," in 2015 IEEE Eindhoven PowerTech, June 2015, pp. 1-5.

[9] B. Lundstrom et al., "Trans-oceanic remote power hardware-in-theloop: multi-site hardware, integrated controller, and electric network cosimulation," IET GTD, vol. 11, no. 18, pp. 4688-4701, 2017.

[10] A. Monti et al., "A global real-time superlab: Enabling high penetration of power electronics in the electric grid," IEEE Power Electronics Magazine, vol. 5, no. 3, pp. 35-44, Sep. 2018.

[11] M. Mirz et al., "Dynamic phasors to enable distributed real-time simulation," in 2017 ICCEP, June 2017, pp. 139-144

[12] K. G. Ravikumar et al., "Distributed simulation of power systems using real-time digital simulator," in 2009 IEEE/PES Power Systems Conference and Exposition, March 2009, pp. 1-6.

[13] M. Stevic et al., "Virtual integration of laboratories over long distance for real-time co-simulation of power systems," in IECON 2016, Oct 2016, pp. 6717-6721.

[14] —, "Empirical study of simulation fidelity in geographically distributed real-time simulations," in 2017 NAPS, Sep. 2017, pp. 1-6.

[15] _ _ "From monolithic to geographically distributed simulation of hydc systems," in 2018 IEEE 19th Workshop on Control and Modeling for Power Electronics (COMPEL), June 2018, pp. 1-5.

[16] M. Mirz et al., "Distributed real-time co-simulation as a service," in 2018 IEEE IESES, Jan 2018, pp. 534-539.

[17] M. Stevic et al., "A bilateral teleoperation approach for interface algorithms in distributed real-time simulations," in 2018 IEEE Workshop on Complexity in Engineering (COMPENG), Oct 2018, pp. 1-5.

[18] S. Vogel et al., "Distributed real-time simulation and its applications to wind energy research," in 2018 IEEE International Conference on Probabilistic Methods Applied to Power Systems (PMAPS), June 2018 pp. 1-6.

[19] M. Stevic et al., "Multi-site european framework for real-time cosimulation of power systems," IET GTD, vol. 11, no. 17, pp. 4126-4135, 2017.

[20] R. Liu et al., "Geographically distributed real-time digital simulations using linear prediction," International Journal of Electrical Power \& Energy Systems, vol. 84, pp. 308 - 317, 2017.

[21] S. Vogel et al., "Improvements to the co-simulation interface for geographically distributed real-time simulationmulti-site european framework for real-time co-simulation of power systems," in IECON 2019, vol. 1, Oct 2019, pp. 6655-6662.

[22] E. Bompard et al., "A multi-site real-time co-simulation platform for the testing of control strategies of distributed storage and $\mathrm{v} 2 \mathrm{~g}$ in distribution networks," in 2016 18th European Conference on Power Electronics and Applications (EPE'16 ECCE Europe), Sep. 2016, pp. 1-9.

[23] A. Estebsari et al., "An iot realization in an interdepartmental real time simulation lab for distribution system control and management studies," in 2016 IEEE 16th International Conference on Environment and Electrical Engineering (EEEIC), June 2016, pp. 1-6.

[24] J. Weimer et al., "A virtual laboratory for micro-grid information and communication infrastructures," in 2012 3rd IEEE PES Innovative Smart Grid Technologies Europe (ISGT Europe), Oct 2012, pp. 1-6.

[25] Y. Wang et al., "A distributed control scheme of microgrids in energy internet and its multi-site implementation," IEEE Trans. on Industrial Informatics, vol. 66, no. 1, pp. 1-1, Feb 2020.

[26] L. Pellegrino et al., "Remote Laboratory Testing Demonstration," Energies, vol. 13, no. 9, p. 2283, May 2020.

[27] B. Palmintier et al., "A power hardware-in-the-loop platform with remote distribution circuit cosimulation," IEEE Trans. on Ind. Electron., vol. 62, no. 4, pp. 2236-2245, April 2015.

[28] Vogel, S. et al., "Distributed Power Hardware-in-the-Loop Testing Using a Grid-Forming Converter as Power Interface," Energies, vol. 13, no. 15, p. 3770 , Jul 2020 .

[29] L. Pellegrino et al., Laboratory Coupling Approach. Springer International Publishing, 2020, pp. 67-86.

[30] R. Brandl, "Operational range of several interface algorithms for different power hardware-in-the-loop setups," Energies, vol. 10, no. 12, p. 1946, Nov 2017.
[31] J. Wu et al., "Generalized three phase coupling method for distributed simulation," in Proceedings of the 37th Annual North American Power Symposium, Nov 2005, pp. 1-6.

[32] Q. Huang et al., "Distributed simulation applied to shipboard power systems," in 2007 IEEE ESTS, May 2007, pp. 498-503.

[33] J. Wu et al., "Distributed simulation for power system analysis including shipboard systems," Electric Power Systems Research, vol. 77, no. 8, pp. 1124 - 1131, 2007

[34] J. L. Bastos et al., "Distributed simulation using the virtual test bed and its real-time extension," in Proceedings of the 2007 Summer Computer Simulation Conference, SCSC 2007, San Diego, California, USA, July 16-19, 2007, 2007, pp. 757-765.

[35] W. Ren et al., "Improve the stability and the accuracy of power hardware-in-the-loop simulation by selecting appropriate interface algorithms," IEEE Trans. Ind. Appl., vol. 44, no. 4, pp. 1286-1294, 2008.

[36] Steffen Vogel, "Development of a modular and fully-digital PCIebased interface to Real-Time Digital Simulator," Master's thesis, RWTH Aachen University, 2017.

[37] M. Syed et al., "A Synchronous Reference Frame Interface for Geographically Distributed Real-Time Simulations," IET GTD, pp. 1-11, 2020.

[38] GÉANT. Géant - at the heart of research and education networking. [Online]. Available: https://www.geant.org/About

[39] _. Géant topology map. [Online]. Available: https://www.geant.org/ Networks/Pan-European network/Pages/GEANT topology map.aspx

[40] ESnet. About esnet. [Online]. Available: https://www.es.net/about/

[41] - Esnet map. [Online]. Available: https://www.es.net/assets/ About-ESnet/15-CS-1035-ESnet-EuropeUS-MapEEXv4.pdf

[42] C. Wiezorek et al., "Multi-location virtual smart grid laboratory with testbed for analysis of secure communication and remote co-simulation: concept and application to integration of berlin, stockholm, helsinki," IET GTD, vol. 11, no. 12, pp. 3134-3143, 2017

[43] V. Jacobson et al. Rtp: A transport protocol for real-time applications. [Online]. Available: https://tools.ietf.org/html/rfc3550

[44] S. Vogel et al., "An open solution for next-generation real-time power system simulation," in 2017 IEEE Conference on Energy Internet and Energy System Integration (EI2), Nov 2017, pp. 1-6.

[45] A. Srivastava et al., "Modeling Cyber-Physical Vulnerability of the Smart Grid With Incomplete Information," IEEE Trans. on Smart Grid, vol. 4, no. 1, pp. 235-244, 2013.

[46] A. Bindra, "Securing the Power Grid: Protecting Smart Grids and Connected Power Systems from Cyberattacks," IEEE Power Elect. Mag., vol. 4, no. 3, pp. 20-27, 2017

[47] E. Guillo-Sansano et al., "Characterization of time delay in power hardware in the loop setups," IEEE Trans. on Ind. Electron., 12020.

[48] W. Ren et al., "Interfacing issues in real-time digital simulators," IEEE Trans. on Power Delivery, vol. 26, no. 2, pp. 1221-1230, April 2011.

[49] E. Guillo-Sansano et al., "Harmonic-by-harmonic time delay compensation method for phil simulation of low impedance power systems," 2015 International Symposium on Smart Electric Distribution Systems and Technologies (EDST), pp. 560-565, 2015.

[50] _ " "Initialization and synchronization of power hardware-in-the-loop simulations: A great britain network case study," Energies 2018, vol. 11, no. 5, p. 1087, 2018.

[51] M. Maniatopoulos et al., "Combined control and power hardware inthe-loop simulation for testing smart grid control algorithms," IET GTD vol. 11, no. 12, pp. 3009-3018, 2017.

[52] S. Chatzivasileiadis, D. Ernst, and G. Andersson, "Chapter 14 - Global power grids for harnessing world renewable energy," in Renewable Energy Integration, 2nd ed., L. E. Jones, Ed. Boston: Academic Press, 2017, pp. $161-174$

[53] C. D. López et al., "Speeding up ac circuit co-simulations through selective simulator decoupling of predictable states," IEEE Access, vol. 7, pp. $43004-43017,2019$.

[54] P. Baumann et al., "Using the distributed co-simulation protocol for a mixed real-virtual prototype," in 2019 IEEE International Conference on Mechatronics (ICM), vol. 1, March 2019, pp. 440-445. 


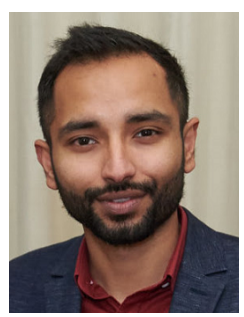

Mazheruddin H. Syed (GS'11-M'18) received his $\mathrm{BE}$ degree in electrical and electronics engineering from Osmania University, India, his MSc degree in electrical power engineering from Masdar Institute of Science and Technology, UAE, and his PhD degree in electrical power systems from the University of Strathclyde, Glasgow, Scotland. Currently, he is a Research Fellow with the Institute for Energy and Environment, University of Strathclyde, serves as the manager for the Dynamic Power Systems Laboratory Microgrid facility and a chartered engineer. He is active in a number of national and international committees, has contributed to innovative power system research projects with a strong publication record His research interests include demand side management, decentralized and distributed control, real-time controller and power hardware in the loop simulations, geographically distributed simulations and systems level validations.

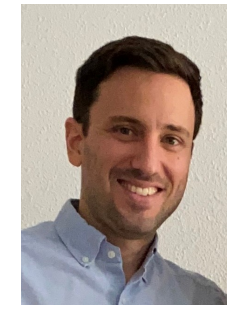

Efren Guillo-Sansano (GS'13-M'18) received his M.Sc. and PhD degrees in Electrical Power Engineering from the University of Strathclyde, Glasgow, UK, in 2013 and 2018. He is currently a Research Associate with the Institute for Energy and Environment in the Department of Electronic and Electrical Engineering. He is a technical lead for the control and power hardware integration and validation at the Dynamic Power Systems Laboratory. His current research interests include the development and validation of distributed energy resources and power electronics, power hardware in the loop, distributed control, and ancillary services.

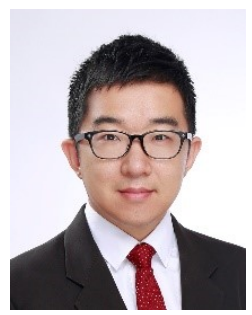

Yu Wang (S'12-M'17) received the B.Eng. degree in Electrical Engineering and Automation from Wuhan University, Wuhan China in 2011, and the M.Sc. and $\mathrm{Ph} . \mathrm{D}$. degree in Power Engineering from Nanyang Technological University, Singapore in 2012 and 2017 , respectively. He is currently a research fellow in Rolls-Royce@NTU Corporate Lab, Singapore. He is working on multiple research/industry projects on microgrids, power systems and energy storage systems. He is an active researcher and reviewer in power and energy engineering society, and is the associate editor of IET Smart Grid. His research interests include distributed control and optimization for power systems and microgrids.

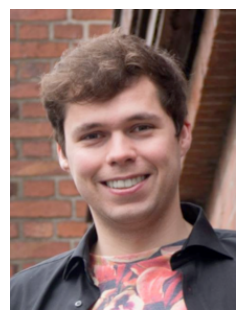

Steffen Vogel (stvogel@eonerc.rwth-aachen.de) received his B.Sc. and M.Sc. degrees in electrical engineering, information technology, and computer engineering in 2014 and 2017, respectively, both from RWTH Aachen University, Germany. Currently, he is a researcher at the Institute for Automation of Complex Power Systems, Rheinisch-Westfälische Technische Hochschule Aachen University, Aachen, Germany.

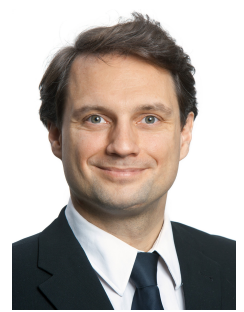

Peter Palensky received his MSc. in Electrical Engineering in 1997 his $\mathrm{PhD}$ in 2001, and his Habilitation in 2015, all from the Vienna University of Technology, Austria. He co-founded a Envidatec, a German startup on energy management and analytics, and joined the Lawrence Berkely National Laboratory, CA, as researcher, and the University of Pretoria, South Africa, in 2008. In 2009 he became appointed head of business unit on sustainable building technologies at the Austrian Institute of Technology (AIT), and later the first Principle Scientist for complex energy systems at the AIT. In 2014 he was appointed full Professor for intelligent electric power grids at TU Delft. He is active in international committees like ISO or CEN and serves an IEEE IES AdCom member-at-large in various functions for the IEEE. He is editor in chief of the IEEE Industrial Electronics Magazine, associate editor for several other IEEE publications, and regularly organizes IEEE conferences. His main research fields are energy automation net-works, smart grids, and modeling intelligent energy systems

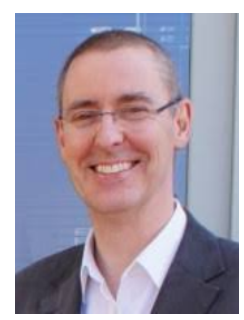

Graeme M. Burt (M'95) received the B.Eng. degree in electrical and electronic engineering, and the $\mathrm{Ph} . \mathrm{D}$. degree in fault diagnostics in power system networks from the University of Strathclyde, Glasgow, U.K., in 1988 and 1992, respectively. He is currently a Professor of electrical power systems at the University of Strathclyde where he co-directs the Institute for Energy and Environment, directs the Rolls-Royce University Technology Centre in Electrical Power Systems, and is lead academic for the Power Networks Demonstration Centre (PNDC). In addition, he serves as spokesperson for the board of DERlab e.V., the association of distributed energy laboratories. His research interests include the areas of power system protection and control, distributed energy, hybrid propulsion and experimental validation.

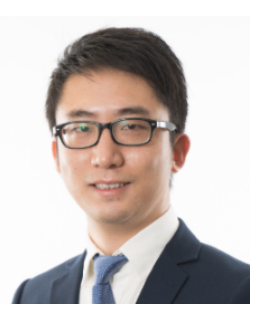

Yan Xu (S'10-M'13-SM'19) received the B.E. and M.E degrees from South China University of Technology, Guangzhou, China in 2008 and 2011, respectively, and the Ph.D. degree from The University of Newcastle, Australia, in 2013. He is now the Nanyang Assistant Professor at School of Electrical and Electronic Engineering, Nanyang Technological University (NTU), and a Cluster Director at Energy Research Institute@ NTU (ERI@N), Singapore. Previously, he held The University of Sydney Postdoctoral Fellowship in Australia. His research interests include power system stability and control, microgrid, and data-analytics for smart grid applications. $\mathrm{Dr} \mathrm{Xu}$ is an Editor for IEEE TRANSACTIONS ON SMART GRID, IEEE TRANSACTIONS ON POWER SYSTEMS, IEEE POWER ENGINEERING LETTERS, and an Associate Editor for IET Generation, Transmission Distribution, IET Energy Conversion Economics, and CSEE Journal of Power and Energy Systems.

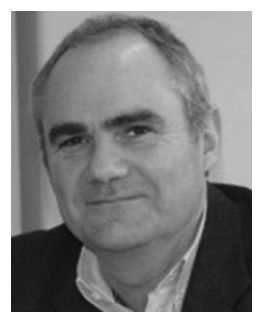

Antonello Monti received the M.Sc. degree (summa cum laude) and the Ph.D. degree in electrical engineering from the Politecnico di Milano, Milan, Italy, in 1989 and 1994, respectively.,He started his career in Ansaldo Industria, Milan, and then moved to the Politecnico di Milano, in 1995, as an Assistant Professor. In 2000, he joined the Department of Electrical Engineering, University of South Carolina, Columbia, SC, USA, as an Associate Professor and then a Full Professor. Since 2008, he has been the Director of the E.ON Energy Research Center, Institute for Automation of Complex Power System, RWTH Aachen University, Aachen, Germany. He has authored or coauthored over 300 peerreviewed articles published in international journals and in the proceedings of international conferences.,Dr. Monti was a recipient of the 2017 IEEE Innovation in Societal Infrastructure Award. $\mathrm{He}$ is an Associate Editor of IEEE System Journal and IEEE Electrification Magazine.

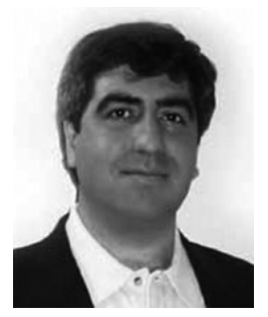

Rob Hovsapian received the M.S. degree in controls and the Ph.D. degree in energy systems from the Mechanical Engineering Department, Florida State University, Tallahassee, FL, USA, in 1988 and 2009, respectively..,He has spent more than 20 years working with Idaho National Laboratory, General Dynamics, TRW, and Northrop Grumman, as a Research Faculty with the Mechanical Engineering Department, Florida State University, and as a Program Manager with the Office of the Naval Research, Center for Advanced Power Systems for the Electrical Ship Research and Development Consortium, Florida State University. $\mathrm{He}$ is currently working with the National Renewable Energy Laboratory, Golden, CO, USA, as a Research Advisor. He has number of publications in the field of energy systems, thermodynamics optimization, thermal modeling, wind energy, and controls. 